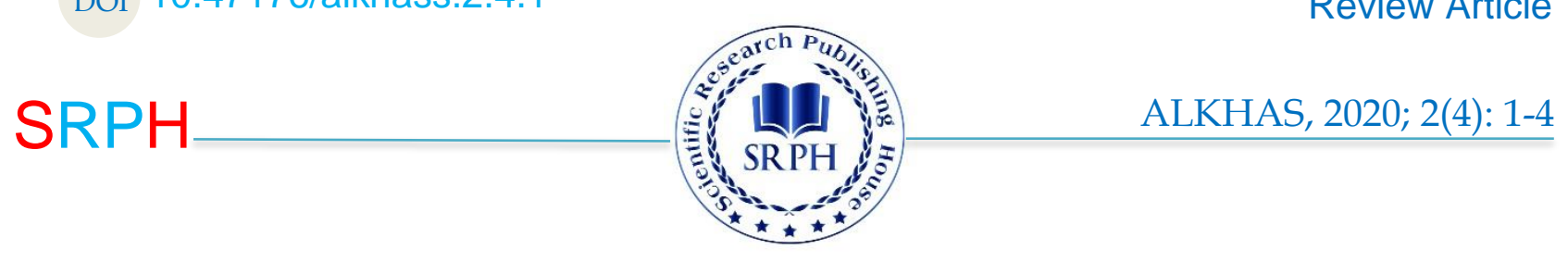

\title{
A Review on the Effect of Natural Antioxidants (Extracts and Nano Extracts) on the Oxidative Stability of Vegetable Oils
}

\author{
Fereshteh Fatourehchi*
}

Zar Macaron University of Applied Sciences and Technology, Iran.

\section{*Corresponding Author: \\ $\rtimes$ fatoorechi.f@gmail.com}

Received: 15 September, 2020

Accepted: 30 October, 2020

Published: 15 December, 2020

\begin{abstract}
One of the major problems facing the food industry is the oxidation of fats and oils, which results in the formation of free radicals. Free radicals cause diseases such as cancer, premature aging and atherosclerosis, and in addition to endangering consumer health, they reduce the nutritional and digestive value of foods. One of the most effective ways to delay lipid oxidation is the use of antioxidants. Natural antioxidants, such as herbal extracts, are an abundant and remarkable source of polyphenolic compounds that have high antioxidant capacity and can be a good alternative to synthetic antioxidants in the food system. Since plant extracts are highly active compounds, they may lose their beneficial effects due to exposure to oxygen or nutrients. Therefore, it is necessary to use methods to protect them in order to achieve the highest antioxidant activity. So, using nano-encapsulation these problems will be partially solved. This paper will review the oxidative of vegetable oils and ways to combat them.
\end{abstract}

Keywords: Oil, Oxidation, Extract, Nano Extract

\section{Introduction}

Oils and fats provide a significant portion of the energy required by the body, essential fatty acids, and fatsoluble vitamins [1]. Oils and fats are obtained from various plant and animal sources, each with different metabolic, physical and chemical properties. Due to the significant number of double bonds in oils, these materials are prone to oxidative corruption [2].

Natural antioxidants extracted from herbals are among the most important bioactive compounds that can be used in food production. Polyphenolic compounds are secondary metabolites of herbals and are one of the most important antioxidant compounds that can be used in the production of beneficial foods or to prevent oxidation and increase the shelf life of oxygen-sensitive foods. In herbals, these compounds are derived from ephenylalanine and include insoluble types such as tannins, lignin, cell wall-bound hydrocyanic acid, or soluble compounds including Phenolic acid, Phenyl Propanoid, Flavonoid, and Quinone [3].

Giving the fact that plant extracts have antioxidant activity due to the presence of active compounds such as Polyphenols, so researchers have studied their use to stabilize edible oils under storage and heating conditions for years. However, problems such as nondissolution of extracts in oil due to their polar nature, consumption of large amounts of extracts to achieve stability and change in color and taste of oil, has attracted the attention of researchers to methods such as Nano emulsions and Nano encapsulating [4].

Therefore, in the following, we will discuss and examine a review regarding the effect of extracts and Nano-extracts on oil shelf life.

\section{Oxidation of oil}

Fats and oils are valuable foods that in addition to providing energy, they play an important role in survival and health of body and are in the group of essential consumer goods. The value and importance of oils in the human diet has a significant role and in addition to the highest level of energizing compared to other food groups, it is a good solvent of vitamins A, $\mathrm{D}, \mathrm{E}$ and $\mathrm{K}$. More over, they are being an energy source for the human body and a supplier of essential fatty acids such as linolenic acid which the body is unable to make it. Most fats and vegetable oils are 
consumed orally after removing impurities and performing the necessary processes [5]. Oils are prone to oxidation and spoilage due to the presence of significant amounts of double bonds. Oxidation is one of the most important and well-known causes of lipid spoilage during oil storage or processing. Most vegetable oils contain large amounts of unsaturated fatty acids that are highly susceptible to oxidation. This process is not only associated with the development of spicy taste, unpleasant aroma and paleness, but also reduces the nutritional value of oils and fats [6]. In order to improve the quality of oil, increase its stability and duration of use, various methods are performed by several companies in the world which include: a mixture of different oils, research and use of new plant species to produce better stable oils and to use synthetic and natural antioxidants [7].

To maintain fats during storage, synthetic antioxidants such as BHA (Butylated hydroxyanisole), BHT (Butylated hydroxytoluene), TBHQ (tert-butyl hydroquinone) and PG (Propyl gallate) are used as food additives to protect food against spoilage [8]. There is ample evidence to support the toxicity and malnutrition effects of synthetic antioxidants added to food. In addition, the risk of liver damage and cancer in laboratory animals is one of the disadvantages of using synthetic antioxidants [9]. Given this situation, currently the most appropriate way to prevent the use of synthetic antioxidants is to find plant sources that contain high level of natural antioxidants such as simple Phenols, Phenolic acids, Carotenoids, Anthocyanins, Flavonoids, vitamins and spice extracts and they have no problem in terms of being found in different geographical areas and climates; it means that they should be easily accessible $[10,11]$.

\section{Natural antioxidants}

Antioxidant compounds in foods can increase the shelf life of processed or unprocessed foods. The use of plant extracts instead of chemical preservatives reduces the concerns of consuming such substances and consumers are more inclined to use these natural preservatives, Plant extracts and their compounds have been used as flavorings since ancient times and now, it has been shown that they have a wide range of antioxidant activities [5]. The antioxidant properties of plant extracts mainly depend on the phenolic compounds in them, but the type of phenolic compounds and consequently their retention power are different in different plants [10]. The use of natural antioxidants or the design of food formulations in such a way that its antioxidant properties are considered to provide part of the needs of modern man. In recent years, natural foods and antioxidant compounds of plant extracts such as vitamins and phenolic compounds have received increasing attention due to their beneficial effects on human health and prevention of oxidative damage. Recently, the use of phenolic compounds in plants has increased due to their potential antioxidant properties and health effects, especially when these compounds are present in high amounts in food. It has also been proven that the origin of many drugs and therapeutic substances is due to secondary metabolism in plants that phenolic compounds with antioxidant and medicinal properties are among the secondary metabolites of plants [12].

Polyphenols have ideal structural properties for their antioxidant activity, mainly due to their ability to donate hydrogen atoms or electrons. Many phenolic compounds of natural origin are remarkable for their properties. However, in free (non-encapsulated) form, they have limited solubility in water. In addition, many polyphenols have an unpleasant taste, which must be covered with oral medications before being added to food [13] .

\section{Micro encapsulation}

One of the methods of preserving active foods such as oils and antioxidant compounds is micro encapsulation. Micro encapsulation is a very effective technique that separates reactive materials from other components of the food system and controls their release. This method leads to the protection of these compounds from environmental damage. Micro encapsulation is a technique in which the desired composition is trapped and encapsulated with the help of one or more materials. Microencapsulation technology began in the 1950s and has developed extensively today and has found many applications in various industries such as pharmaceutical, chemical and food industries and printing [14]. The most important criteria in selecting a suitable material for encapsulation include the ability that the encapsulated material can provide for the final product. Potential limitations for the encapsulated material, concentration of encapsulated material, type of active substance and its characteristics and application, type of release, Sustainability requirements and cost constraints. The materials used to design the encapsulating protective shell must be food grade and permitted for food applications as generally recognized as safe, biodegradable and capable of forming a barrier between the internal and surrounding phases. Most of the materials used for encapsulation in the food industry are biomolecules. In addition to being natural, the materials used should provide maximum protection of the active substance against environmental conditions, maintain the active phase during the process by holding under different conditions inside the capsule structure, do not react with the encapsulated material, if necessary, they should have good rheological properties and be able to work comfortably during encapsulation [15]. 


\section{A review of studies}

Chatterjee and Bhattacharjee [16] investigated the effect of clove extract and nano-capsule clove extract on the stability of soybean oil and compared it with BHT synthetic antioxidant during storage and thermal conditions. They reported that clove nanocapsule extract as a natural antioxidant could hopefully replace synthetic antioxidants in the food industry.

Hasani and Javadian [17] in connection with the addition of nano-capsule extract of orange peel on carp fillets stated that increasing the concentration of the extract and also the use of nano-capsule extract slows down the changes in peroxide number during storage. Also, in these treatments, the least amount of fat oxidation occurs.

Bakhshandeh et al. [18] investigated the effect of free and nano-encapsulated cannabis extracts on the oxidative stability of soybean oil. The results showed that in both methods, the antioxidant activity of the extract increased with increasing the concentration of the extract. Hemp extract was added to the encapsulated material in a ratio of 1: 5 . The size of the capsules varied between 150.71 to $171.42 \mathrm{~nm}$. According to the results, oils containing nanoencapsulated Hemp extract had the lowest amount of fat oxidation during storage compared to samples containing TBHQ and pure extract, and samples containing oil encapsulated with Maltodextrin: Chitosan extract had the lowest oxidation indices (Peroxide value and Thiobarbituric acid number).

\section{Conclusion}

Oxidation of vegetable oils is a major challenge in the food industry, resulting in major economic losses and reduced nutritional quality of fatty foods. Preservatives are used to increase the shelf life and slow down the oxidation of the oil. The use of chemical preservatives poses a serious threat to consumer health. Due to the desire of consumers to use natural preservatives, the need to use herbal extracts that have antioxidant and antimicrobial properties is well felt. On the other hand, not dissolving the extracts well in the oil and uncontrolled release of phenols in the extract will reduce its antioxidant efficiency, so using Nano capsulation will solve these problems to some extent. By reviewing of the researches conducted in this study, the usefulness of using the Nano capsule extract was confirmed.

\section{References}

1. Keratin M, Hosseini Mazarin SZ, Gourami A, Ghazis B, Esfandyari Ch. Survey on the rate of losses and wastes in oil seeds processing plants in Tehran province. Food Technol Nutr. 2011; 8: 42-50.
2. Santas J, Guzman Y, Guardiola F, Rafecas M, Bou R. High-throughput analysis of lipid hydroperoxides in edible oils and fats vsing the fluorescent reagent diphenyl-1-pyrenylphosphine. Food Chem. 2014; 29: 235-2410.

3. Sariri R. Antioxidant activity exhibited by medicinal plants, vegetables and fruits from North of Iran. Res Signpost. 2012; 37/661(2): 205-236.

4. Khazaei KM, Jafari S, Ghorbani M, Kakhki AH. Application of maltodextrin and gum Arabic in microencapsulation of saffron petals anthocyanins and evaluating their storage stability and color. Carbohydr polymer. 2014; 105: 57-62.

5. Inanc T, Maskan M. The potential application of plant essential oils/extracts as natural preservatives in oils during processing: A review. J Food Sci Eng. 2012; 2: 1-9.

6. Zhang Y, Yang L, Zu Y, Chen X, Wang F, Liu F. Oxidative stability of sunflower oil supplemented with carnosic acid compared with synthetic antioxidants during accelerated storage. Food Chem. 2010; 118: 656662.

7. Chammem N, Saoudi S, Sifaoui I, Sifi S, Person MD, Abderraba M, Moussa F, Hamdi M. Improvement of vegetable oils quality in frying conditions by adding rosemary extract. Ind Crop Prod. 2015; 14: 512-511.

8. Tawaha K, Alali FQ, Gharaibeh M, Mohammmad M, EL-Elimat T. Antioxidant activity and total phenolic content of selected Jordanian plant species. Food Chem. 2007; 104: 1372-1378.

9. Ding M, Zou J. Rapid micropreparation procedure for the gas chromatographic-mass spectrometric determination of BHT, BHA and TBHQ in edible oils. Food Chem. 2012; 131: 1251-1255.

10. Bodoira RM, Penci MC, Ribotta PD, Martínez ML. Chia (Salvia hispanica L.) oil stability: Study of the effect of natural antioxidants, LWT. Food Sci Technol. 2017; 15: 121-113.

11. Wannurul Z. Extraction of antioxidant and compounds from red, pitaya using soxhlet extraction method. Thesis of Faculty of Chemical \& Natural Resources Engineering Universiti Malaysia Pahang. 2009.

12. Salmanian Sh, Sadeghi Mahonak A, Aalmi M, Ghorbani M. Evaluation of antiradical activity of antioxidants and determination of flavonoid compounds of Velik fruit. Iran J Nutr Sci Food Ind. 2012; 8(1): 185-177.

13. Gibbs M, Vogt E, Weiss J. Encpsulation of polyphenolic grape seed extract in polymerencapsulated liposomes. Food Funct. 2012; 3: 246-254.

14. Chaudhry Q, Scotter M, Blackburn J, Ross B, Boxall A, Castle L. Applications and applications of nanotechnologies for the food sector. Food Addit Contam. 2008; 25(3): 241-258.

15. Nedovic V, Manojlovic V, Levic S, Bugarskib B. Anoverview of encapsulation technologies for foodapplications. Procedia Food Sci. 2011; 1: 1806-1815. 
16. Chatterjee D, Bhattacharjee P. Comparative evaluation of the antioxidant efficacy of encapsulated and un-encapsulated eugenol-rich clove extracts in soybean oil: Shelf-life and frying stability of soybean oil. J Food Eng. 2013; 117: 545-550.

17. Hasani O, Javadian SR. Effect of encapsulated bitter orange peel extract and BHT on the quality of common carp fillet during refrigerated storage. Int J Food Eng. 2016; 12(3): 303-310.

18. Bakhshandeh T, Esmaeibzadeh Kenari R, Raftani Amiri Z. The effect of free and nano-encapsulated extract of Hemp on oxidative stability of soybean oil. $J$ Food Sci Technol. 2018; 81(15): 237-249.

\section{ALKHAS}

Copyright: (C) 2020 The Author(s); This is an open-access article distributed under the terms of the Creative Commons Attribution License (http://creativecommons.org/licenses/by/4.0), which permits unrestricted use, distribution, and reproduction in any medium, provided the original work is properly cited.

Citation: Fatourehchi F. A Review on the Effect of Natural Antioxidants (Extracts and Nano Extracts) on the Oxidative Stability of Vegetable Oils. ALKHAS. 2020; 2(4): 1-4.

https://doi.org/10.47176/alkhass.2.4.1 